\title{
Exploring $X(5568)$ as a meson molecule
}

\author{
S. S. Agaev, ${ }^{1,2}$ K. Azizi, ${ }^{3}$ and H. Sundu ${ }^{1}$ \\ ${ }^{1}$ Department of Physics, Kocaeli University, 41380 Izmit, Turkey \\ ${ }^{2}$ Institute for Physical Problems, Baku State University, Az-1148 Baku, Azerbaijan \\ ${ }^{3}$ Department of Physics, Doğuş University, Acibadem-Kadiköy, 34722 Istanbul, Turkey
}

( $\Omega$ Dated: August 28, 2018)

\begin{abstract}
The parameters, i.e. the mass and current coupling of the exotic $X(5568)$ state observed by the D0 Collaboration as well as the decay width of the process $X \rightarrow B_{s}^{0} \pi^{+}$are explored using $B \bar{K}$ molecule assumption on its structure. Employed computational methods include QCD two-point and light-cone sum rules, latter being considered in the soft-meson approximation. The obtained results are compared with the data of the D0 Collaboration as well as with the predictions of the diquark-antidiquark model. This comparison strengthens a diquark-antidiquark picture for the $X(5568)$ state rather than a meson molecule structure.
\end{abstract}

PACS numbers: 14.40.Rt, 12.39.Mk, 11.55.Hx

\section{INTRODUCTION}

Both the experimental and theoretical investigations of exotic hadronic states, i.e. particles that are beyond the schemes of traditional hadron spectroscopy, seem already formed as one of the interesting and rising branches of the high energy physics. Since the pioneering discovery of the charmoniumlike resonance $X(3872)$ by Belle Collaboration in 2003 [1] (see, also Refs. 2-4]), numerous exotic particles were observed and studied. Now they are organized as the $X Y Z$ family of the exotic particles. During passed years, experimental and theoretical investigation of these particles achieved evident successes in measurements of their masses and decay widths, in exploring spins and parities, as well as in creating various theoretical models and schemes to reveal their internal structure and compute corresponding parameters (see for instance [5-12] and references therein).

Recently, due to the observation of the resonance structure reported by the D0 Collaboration in Ref. 13], this interest to unusual hadrons is renewed. Really, in accordance with this observation, the new narrow resonance $X(5568)$ (in what follows denoted as $X_{b}(5568)$ or $X_{b}$ ) contains four valence quarks and belongs to the class of the exotic states. But what is important, the $X_{b}(5568)$ is probably the first hadronic state built of fourquarks of different flavors, namely $b, s, u$ and $d$ quarks, which makes it a very interesting object for the experimental and theoretical studies. The D0 Collaboration measured its mass, width of the dominant decay channel $X_{b} \rightarrow B_{s} \pi$, and assigned the quantum numbers $J^{P C}=0^{++}$to this resonance as preferable ones. Moreover, in Ref. [13] some suggestions concerning the structure of the $X_{b}(5568)$ were made, as well. Thus, the $X_{b}(5568)$ may be considered as a diquark-antidiquark bound state within the tetraquark model of the exotic states. Alternatively, $X_{b}$ may be studied as a molecule composed of $B$ and $\bar{K}$ mesons.

Nevertheless, despite the experimental information on the $X_{b}(5568)$ state provided by the D0 Collaboration, the
LHCb Collaboration has not still confirmed the D0 observation, as they announced in a preliminary report [14]. This situation demands intensive experimental and theoretical explorations of its features to answer questions on the nature of the observed state.

The information of the D0 Collaboration led to burst of activity in the relevant theoretical studies. It started immediately after discovery of the exotic $X_{b}(5568)$ state and employed almost all possible scenarios to explain its observed parameters. In fact, in Refs. [15, 16] we calculated the mass, decay constant and width of the $X_{b}(5568)$ state within the diquark-antidiquark picture $X_{b}=[s u][\bar{b} \bar{d}]$ considering the exotic state with positive charge. Our results for the mass $m_{X_{b}}$, and for the width of its decay $\Gamma\left(X_{b} \rightarrow B_{s}^{0} \pi^{+}\right)$are in a good agreement with the experimental data.

In Ref. [17] we extended our investigation of the new family of the four-quark exotic states by considering the charmed partner of the $X_{b}$ state, and assuming that the $X_{c}$ state is composed of the $c, s, u$ and $d$ quarks. We also supposed that $X_{c}$ possesses the same quantum numbers as $X_{b}$. We computed the mass, decay constant and width of the decays $X_{c} \rightarrow D_{s}^{-} \pi^{+}$and $X_{c} \rightarrow D^{0} K^{0}$ considering $X_{c}$ as $[s u][\bar{c} \bar{d}]$ diquark-antidiquark state and employing two forms for the interpolating currents.

The mass of the $X_{b}$ state was also estimated in Refs. [18 21] using the diquark-antidiquark model. The width of the decay channel $X_{b}^{ \pm}(5568) \rightarrow B_{s} \pi^{ \pm}$was calculated in Ref. 22, 23] in the framework of the three-point QCD sum rule approach, and good agreement between the theoretical predictions for $\Gamma\left(X_{b}^{ \pm} \rightarrow B_{s}^{0} \pi^{ \pm}\right)$and experimental data was reported. As a bound state of the $B$ and $\bar{K}$ mesons the exotic $X_{b}$ particle was studied in Ref. [24]. Some questions of quark-antiquark organization of $X_{b}$ and its partners were addressed in Ref. [25].

The controversial information of the D0 and LHCb collaborations triggered an appearance of interesting theoretical works devoted to analysis of the $X_{b}$ physics. In these papers numerous aspects of the $X_{b}$ problems, including investigations of its structure and parameters, analysis some of production mechanisms are covered. For 
details and further explanations we refer to original works [26 35].

In the present article we continue our investigation of the exotic state $X_{b}$ by supposing that it can be considered as a meson molecule. In other words, we assume that $X_{b}$ is a molecule-like state composed of $B^{+}$and $\bar{K}^{0}$ mesons. We are going to calculate its mass, decay constant and width of the decay channel using the corresponding molecule-type interpolating current and theoretical methods presented in rather detailed form in Refs. [15 17, 36]. Our aim is to answer the question: has the exotic $X_{b}$ state observed by the D0 Collaboration the structure of a meson molecule or it is a tightly-bound tetraquark state?

This work is organized in the following way. In Section [II] we introduce the interpolating current employed in QCD sum rule calculations. Here we derive the sum rules to evaluate the mass, decay constant and width of the decay $X_{b} \rightarrow B_{s} \pi^{+}$. In Sect. [II] we present results of numerical calculations and compare them with experimental data and theoretical predictions. This section contains also our conclusions. Explicit expressions for the spectral density required for computation of the mass and decay constant of the $X_{b}$ state are collected in Appendix A.

\section{THE SUM RULES FOR THE MASS, CURRENT COUPLING AND DECAY WIDTH}

In this section we derive QCD sum rule expressions necessary to calculate the mass, current coupling and width of the $X_{b} \rightarrow B_{s} \pi^{+}$decay employing a moleculetype interpolating current. The ways of calculations have been considered in detailed form in our previous papers, therefore we write down below only expressions that are new and differ from ones presented there.

For calculation of the mass and current coupling we use the two-point QCD sum rule and start from the correlation function

$$
\Pi(p)=i \int d^{4} x e^{i p x}\left\langle 0\left|\mathcal{T}\left\{J^{X_{b}}(x) J^{X_{b} \dagger}(0)\right\}\right| 0\right\rangle,
$$

where $J^{X_{b}}(x)$ is the interpolating current with required quantum numbers. We consider $X_{b}$ state as a particle with the quantum numbers $J^{P}=0^{+}$. In the meson molecule scheme the current $J^{X_{b}}(x)$ is given by

$$
J^{X_{b}}(x)=\left[\bar{d}^{a}(x) \gamma_{5} s^{a}(x)\right]\left[\bar{b}^{b}(x) \gamma_{5} u^{b}(x)\right],
$$

where $a$ and $b$ are color indices.

The standard procedures for deriving QCD sum rules include at first stage computation of the correlation function in terms of the physical degrees of freedom. The final expression, which one uses to get the relevant sum rule, is the Borel transformed form of the function $\Pi^{\text {Phys }}(p)$. In the case under consideration it is given as

$$
\mathcal{B}_{p^{2}} \Pi^{\mathrm{Phys}}(p)=m_{X_{b}}^{2} f_{X_{b}}^{2} e^{-m_{X_{b}}^{2} / M^{2}}+\ldots
$$

The second step is to find the theoretical expression for the same function, $\Pi^{\mathrm{QCD}}(p)$, employing the quark-gluon degrees of freedom. To this end, contracting the quark fields for the correlation function $\Pi^{\mathrm{QCD}}(p)$ we find:

$$
\begin{aligned}
& \Pi^{\mathrm{QCD}}(p)=i \int d^{4} x e^{i p x} \operatorname{Tr}\left[\gamma_{5} S_{s}^{a a^{\prime}}(x) \gamma_{5} S_{d}^{a^{\prime} a}(-x)\right] \\
& \times \operatorname{Tr}\left[\gamma_{5} S_{u}^{b b^{\prime}}(x) \gamma_{5} S_{b}^{b^{\prime} b}(-x)\right]
\end{aligned}
$$

where $S_{q}^{a b}(x)$ and $S_{b}^{a b}(x)$ are the light $(q \equiv u, d$ or $s)$ and $b$-quark propagators, respectively. We choose the light quark propagator $S_{q}^{a b}(x)$ in the form

$$
\begin{aligned}
& S_{q}^{a b}(x)=i \delta_{a b} \frac{\not x}{2 \pi^{2} x^{4}}-\delta_{a b} \frac{m_{q}}{4 \pi^{2} x^{2}}-\delta_{a b} \frac{\langle\bar{q} q\rangle}{12} \\
& +i \delta_{a b} \frac{\not m_{q}\langle\bar{q} q\rangle}{48}-\delta_{a b} \frac{x^{2}}{192}\langle\bar{q} g \sigma G q\rangle+i \delta_{a b} \frac{x^{2} \not m_{q}}{1152}\langle\bar{q} g \sigma G q\rangle \\
& -i \frac{g G_{a b}^{\alpha \beta}}{32 \pi^{2} x^{2}}\left[\not \sigma_{\alpha \beta}+\sigma_{\alpha \beta} \not x\right]-i \delta_{a b} \frac{x^{2} \not g^{2}\langle\bar{q} q\rangle^{2}}{7776} \\
& -\delta_{a b} \frac{x^{4}\langle\bar{q} q\rangle\left\langle g^{2} G^{2}\right\rangle}{27648}+\ldots
\end{aligned}
$$

For the $b$-quark propagator $S_{b}^{a b}(x)$ we employ the expression from Ref. [37]

$$
\begin{aligned}
& S_{b}^{a b}(x)=i \int \frac{d^{4} k}{(2 \pi)^{4}} e^{-i k x}\left\{\frac{\delta_{a b}\left(\not k+m_{b}\right)}{k^{2}-m_{b}^{2}}\right. \\
& -\frac{g G_{a b}^{\alpha \beta}}{4} \frac{\sigma_{\alpha \beta}\left(\not k+m_{b}\right)+\left(\not k+m_{b}\right) \sigma_{\alpha \beta}}{\left(k^{2}-m_{b}^{2}\right)^{2}} \\
& +\frac{g^{2} G^{2}}{12} \delta_{a b} m_{b} \frac{k^{2}+m_{b} \not k}{\left(k^{2}-m_{b}^{2}\right)^{4}}+\frac{g^{3} G^{3}}{48} \delta_{a b} \frac{\left(\not k+m_{b}\right)}{\left(k^{2}-m_{b}^{2}\right)^{6}} \\
& \left.\times\left[\not k\left(k^{2}-3 m_{b}^{2}\right)+2 m_{b}\left(2 k^{2}-m_{b}^{2}\right)\right]\left(\not k+m_{b}\right)+\ldots\right\} .
\end{aligned}
$$

In Eqs. (5) and (6) we use the notations

$$
\begin{aligned}
& G_{a b}^{\alpha \beta}=G_{A}^{\alpha \beta} t_{a b}^{A}, \quad G^{2}=G_{\alpha \beta}^{A} G_{\alpha \beta}^{A}, \\
& G^{3}=f^{A B C} G_{\mu \nu}^{A} G_{\nu \delta}^{B} G_{\delta \mu}^{C},
\end{aligned}
$$

where $a, b=1,2,3$ and $A, B, C=1,2 \ldots 8$ are the color indices, and $t^{A}=\lambda^{A} / 2$ with $\lambda^{A}$ being the Gell-Mann matrices. In the nonperturbative terms the gluon field strength tensor $G_{\alpha \beta}^{A} \equiv G_{\alpha \beta}^{A}(0)$ is fixed at $x=0$.

The correlation function $\Pi^{\mathrm{QCD}}\left(p^{2}\right)$ is given by a simple dispersion integral

$$
\Pi^{\mathrm{QCD}}\left(p^{2}\right)=\int_{\left(m_{b}+m_{s}\right)^{2}}^{\infty} \frac{\rho^{\mathrm{QCD}}(s)}{s-p^{2}}+\ldots
$$

where $\rho^{\mathrm{QCD}}(s)$ is the corresponding spectral density. We have calculated the spectral density by including into analysis the quark, gluon and mixed condensates up to eight dimensions. Our result for $\rho^{\mathrm{QCD}}(s)$ is moved to Appendix A. 
Applying the Borel transformation on the variable $p^{2}$ to the invariant amplitude $\Pi^{\mathrm{QCD}}\left(p^{2}\right)$, equating the obtained expression to $\mathcal{B}_{p^{2}} \Pi^{\text {Phys }}(p)$, and subtracting the continuum contribution, we finally obtain the required sum rules. Thus, the mass of the $X_{b}$ state can be evaluated from the sum rule

$$
m_{X_{b}}^{2}=\frac{\int_{\left(m_{b}+m_{s}\right)^{2}}^{s_{0}} d s s \rho^{\mathrm{QCD}}(s) e^{-s / M^{2}}}{\int_{\left(m_{b}+m_{s}\right)^{2}}^{s_{0}} d s \rho^{\mathrm{QCD}}(s) e^{-s / M^{2}}},
$$

whereas for the decay constant $f_{X_{b}}$ we employ the formula

$$
f_{X_{b}}^{2} m_{X_{b}}^{2} e^{-m_{X_{b}}^{2} / M^{2}}=\int_{\left(m_{b}+m_{s}\right)^{2}}^{s_{0}} d s \rho^{\mathrm{QCD}}(s) e^{-s / M^{2}} .
$$

In order to find the width of $X_{b} \rightarrow B_{s} \pi$ decay, we start from calculation of the strong coupling $g_{X_{b} B_{s} \pi}$ using QCD sum rule on the light-cone and soft-meson approximation. We consider the correlation function

$$
\Pi(p, q)=i \int d^{4} x e^{i p x}\left\langle\pi(q)\left|\mathcal{T}\left\{J^{B_{s}}(x) J^{X_{b} \dagger}(0)\right\}\right| 0\right\rangle,
$$

that allows us to get the sum rule for the coupling $g_{X_{b} B_{s} \pi}$. Here the interpolating current $J^{B_{s}}(x)$ is defined in the form:

$$
J^{B_{s}}(x)=\bar{b}_{l}(x) i \gamma_{5} s_{l}(x) .
$$

In the soft-meson limit $q=0$, for the Borel transformed form of the correlation function $\Pi^{\text {Phys }}(p, q=0)$, we get (see, Refs. [16, 36]),

$$
\begin{aligned}
& \Pi^{\mathrm{Phys}}\left(M^{2}\right)=\frac{f_{B_{s}} f_{X_{b}} m_{X_{b}} m_{B_{s}}^{2} g_{X_{b} B_{s} \pi}}{\left(m_{b}+m_{s}\right)} m^{2} \\
& \times \frac{1}{M^{2}} e^{-m^{2} / M^{2}} .
\end{aligned}
$$

where $m^{2}=\left(m_{X_{b}}^{2}+m_{B_{s}}^{2}\right) / 2$.

To proceed, we have to calculate $\Pi^{\mathrm{QCD}}(p, q)$ in terms of the quark-gluon degrees of freedom and find QCD side of the sum rule. Contractions of $s$ and $b$-quark fields in Eq. (11) yield

$$
\begin{aligned}
& \Pi^{\mathrm{QCD}}(p, q)=-\int d^{4} x e^{i p x}\left[\gamma_{5} S_{b}^{b i}(-x) \gamma_{5}\right. \\
& \left.\times S_{s}^{i a}(x) \gamma_{5}\right]_{\alpha \beta}\left\langle\pi(q)\left|\bar{u}_{\alpha}^{b}(0) d_{\beta}^{a}(0)\right| 0\right\rangle,
\end{aligned}
$$

where $\alpha$ and $\beta$ are the spinor indices. Now we use the expansion

$$
\bar{u}_{\alpha}^{b} d_{\beta}^{a} \rightarrow \frac{1}{4} \Gamma_{\beta \alpha}^{j}\left(\bar{u}^{b} \Gamma^{j} d^{a}\right),
$$

where $\Gamma^{j}$ is the full set of Dirac matrixes

$$
\Gamma^{j}=\mathbf{1}, \gamma_{5}, \gamma_{\lambda}, i \gamma_{5} \gamma_{\lambda}, \sigma_{\lambda \rho} / \sqrt{2},
$$

and determine the required local matrix elements. For this purpose, we first perform summation over the color indices. To clarify the computational scheme, let us take a term $\sim \delta_{b i}$ from the b-quark propagator and terms $\sim \delta_{i a}$ from the s-quark propagator considering by this way, terms without gluon contributions. Then we get

$$
\delta_{b i} \delta_{i a} \bar{u}_{\alpha}^{b} d_{\beta}^{a}=\delta_{b a} \frac{\delta_{b a}}{3} \bar{u}_{\alpha} d_{\beta}=\bar{u}_{\alpha} d_{\beta} .
$$

Stated differently, after color summation in these terms we have to use the replacement

$$
\bar{u}_{\alpha}^{b} d_{\beta}^{a} \Rightarrow \bar{u}_{\alpha} d_{\beta} .
$$

Now let us consider contributions $\sim G$. We may take a part $\sim \delta$ from one propagator and nonperturbative part from the another one. Then, as an example, for the color structure of such term we find

$$
\delta_{b i} g G_{a i}^{\varkappa \omega} \bar{u}_{\alpha}^{b} d_{\beta}^{a}=g G_{b a}^{\varkappa \omega} \bar{u}_{\alpha}^{b} d_{\beta}^{a}=g \bar{u}_{\alpha} G^{\varkappa \omega} d_{\beta} .
$$

Therefore, we get a rule

$$
\delta_{b i} g G_{a i}^{\varkappa \omega} \bar{u}_{\alpha}^{b} d_{\beta}^{a} \Rightarrow g\left(\bar{u} G^{\varkappa \omega} d\right) .
$$

This rule allows us to insert into quark matrix elements the gluon field strength tensor $G$ that effectively leads to three-particle components and corresponding matrix elements of the pion: in the present work we neglect terms $\sim G^{2}$ and $\sim G^{3}$. As is seen, in the case of the molecule current the color summation is trivial. One needs only to remove color factors from the propagators and use the prescriptions given above.

Omitting technical details, which can be found in Refs. [16, 36], we provide final expression for the spectral density, which is given as a sum of the perturbative and nonperturbative components

$$
\rho_{\mathrm{c}}^{\mathrm{QCD}}(s)=\rho_{\mathrm{c}}^{\text {pert. }}(s)+\rho_{\mathrm{c}}^{\text {n. }-\mathrm{p} \cdot}(s) .
$$

where

$$
\rho_{\mathrm{c}}^{\text {pert. }}(s)=\frac{f_{\pi} \mu_{\pi}}{32 \pi^{2}}\left[s-2 m_{b}\left(m_{b}-m_{s}\right)\right] \sqrt{1-\frac{4 m_{b}^{2}}{s}},
$$

and

$$
\begin{aligned}
& \rho_{\mathrm{c}}^{\text {n. }-\mathrm{p} .}(s)=\frac{f_{\pi} \mu_{\pi}}{24}\langle\bar{s} s\rangle\left[s m_{s} \delta^{(1)}\left(s-m_{b}^{2}\right)-2 m_{b} \delta\left(s-m_{b}^{2}\right)\right] \\
& +\frac{f_{\pi} \mu_{\pi}}{144}\langle\bar{s} g \sigma G s\rangle\left\{6\left(m_{b}-m_{s}\right) \delta^{(1)}\left(s-m_{b}^{2}\right)+3 s\left(m_{b}-2 m_{s}\right)\right. \\
& \left.\times \delta^{(2)}\left(s-m_{b}^{2}\right)-s^{2} m_{s} \delta^{(3)}\left(s-m_{b}^{2}\right)\right\} .
\end{aligned}
$$

In Eq. (21) $\delta^{(n)}\left(s-m_{b}^{2}\right)=(d / d s)^{n} \delta\left(s-m_{b}^{2}\right)$ that appear when extracting the imaginary part of the pole terms.

As is seen, in the soft limit, the spectral density depends only the parameters $f_{\pi}$ and $\mu_{\pi}$ through the pion's local matrix element

$$
\left\langle 0\left|\bar{d}(0) i \gamma_{5} u(0)\right| \pi(q)\right\rangle=f_{\pi} \mu_{\pi},
$$


where

$$
\mu_{\pi}=\frac{m_{\pi}^{2}}{m_{u}+m_{d}}=-\frac{2\langle\bar{q} q\rangle}{f_{\pi}^{2}}
$$

The continuum subtraction is performed in a standard manner after $\rho^{h}(s) \rightarrow \rho_{c}^{\mathrm{QCD}}(s)$ replacement. Then, the final sum rule to evaluate the strong coupling reads

$$
\begin{aligned}
& g_{X_{b} B_{s} \pi}=\frac{\left(m_{b}+m_{s}\right)}{f_{B_{s}} f_{X_{b}} m_{X_{b}} m_{B_{s}}^{2} m^{2}}\left(1-M^{2} \frac{d}{d M^{2}}\right) M^{2} \\
& \times \int_{\left(m_{b}+m_{s}\right)^{2}}^{s_{0}} d s e^{\left(m^{2}-s\right) / M^{2}} \rho_{c}^{\mathrm{QCD}}(s) .
\end{aligned}
$$

The width of the decay $X_{b} \rightarrow B_{s}^{0} \pi^{+}$can be found applying the standard methods. Our calculations give

$$
\begin{aligned}
& \Gamma\left(X_{b} \rightarrow B_{s}^{0} \pi^{+}\right)=\frac{g_{X_{b} B_{s} \pi}^{2} m_{B_{s}}^{2}}{24 \pi} \lambda\left(m_{X_{b}}, m_{B_{s}}, m_{\pi}\right) \\
& \times\left[1+\frac{\lambda^{2}\left(m_{X_{b}}, m_{B_{s}}, m_{\pi}\right)}{m_{B_{s}}^{2}}\right],
\end{aligned}
$$

where

$$
\lambda(a, b, c)=\frac{\sqrt{a^{4}+b^{4}+c^{4}-2\left(a^{2} b^{2}+a^{2} c^{2}+b^{2} c^{2}\right)}}{2 a} .
$$

Equations (24) and (25) are final expressions that will be used for numerical analysis of the decay channel $X_{b} \rightarrow$ $B_{s}^{0} \pi^{+}$.

\section{NUMERICAL RESULTS AND CONCLUSIONS}

The QCD sum rules derived above contain numerous parameters (see, Table I), which have to be fixed in accordance with the usual procedures. Thus, for numerical computation of the $X_{b}$ state's mass and decay constant we need values of the quark, gluon and mixed condensates. For the various vacuum condensates we use their well known values. In this range the gluon condensate $\left\langle g^{3} G^{3}\right\rangle$ is relatively new parameter, for which we employ the estimate given in Ref. 38. The QCD sum rules contain also $b$ and $s$ quark masses, and depend on $B_{s}$ meson's mass and decay constant $f_{B_{s}}$. In the present work, we choose the mass of the $b$ quark in the $\overline{M S}$ scheme at the scale $\mu=m_{b}$, whereas the decay constant $f_{B_{s}}$ is borrowed from the lattice calculations from Ref. [39].

The expressions evaluated within the QCD sum rule method additionally depend on the continuum threshold and Borel parameters, i.e. on $s_{0}$ and $M^{2}$, respectively. One needs to fix some regions where physical quantities under consideration are practically independent or demonstrate weak dependence on them. To find the working window for the Borel parameter, we require the convergence of the operator product expansion, as well as suppression of the contributions arising from the higher resonances and continuum, in other words exceeding of the pole contribution over the ones coming from the higher dimensional condensates. As a result, for the mass and current coupling calculations we find the range of $M^{2}$ as

$$
4 \mathrm{GeV}^{2} \leq M^{2} \leq 6 \mathrm{GeV}^{2}
$$

The continuum threshold depends on the energy of the first excited state with the same quantum numbers and structure as the particle under consideration. In the case of the exotic states, it is difficult to determine unambiguously this energy level, therefore we have to follow standard recipes adopted in the sum rule computations and vary $s_{0}$ within the following region by considering the resonance of the D0 Collaboration as a ground state

$$
34.5 \mathrm{GeV}^{2} \leq s_{0} \leq 37 \mathrm{GeV}^{2} .
$$

Results of our numerical calculations of $m_{X_{b}}$ and $f_{X_{b}}$ are depicted in Figs. 1 and 2 It is not difficult to conclude that, in the chosen region the dependence of the mass and decay constant on the parameter $M^{2}$ is insignificant. At the same time, obtained predictions depend on the threshold parameter $s_{0}$, which is the main source of the uncertainty of the sum rule computations. Variations of other parameters within allowed limits give rise to errors, as well. The using in the leading order sum rule expression the running quark mass $m_{b}(\mu)$, which in the lack of an information on the next-to-leading order correction necessary to fix the renormalization scheme and scale, also generates ambiguity. We treat the ambiguity arising because of the choice of the renormalization scale $\mu$ as an additional source of the theoretical error, and include its effect to the total error of the sum rule results presented below.

Hence, considering $X_{b}$ as the molecule state composed of the $B$ and $\bar{K}$ mesons, for its mass we obtain

$$
m_{X_{b}}=(5757 \pm 145) \mathrm{MeV},
$$

whereas the experimental value of the D0 Collaboration is equal to

$$
m_{X_{b}}=5567.8 \pm 2.9(\text { stat })_{-1.9}^{+0.9}(\text { syst }) \mathrm{MeV} .
$$

For the decay constant $f_{X_{b}}$ we get

$$
f_{X_{b}}=(0.17 \pm 0.04) \cdot 10^{-2} \mathrm{GeV}^{4} .
$$

The coupling $g_{X_{b} B_{s}} \pi$ extracted from the sum rule expression Eq. (24) reads

$$
g_{X_{b} B_{s} \pi}=(0.50 \pm 0.14) \mathrm{GeV}^{-1} .
$$

As a result, for the width of the decay channel $\Gamma\left(X_{b} \rightarrow\right.$ $\left.B_{s} \pi\right)$ we find

$$
\Gamma\left(X_{b} \rightarrow B_{s} \pi\right)=(33.6 \pm 12.1) \mathrm{MeV},
$$

when the experimental data give

$$
\Gamma\left(X_{b} \rightarrow B_{s} \pi\right)=21.9 \pm 6.4(\text { stat })_{-2.5}^{+5.0}(\text { syst }) \mathrm{MeV} \text {. }
$$




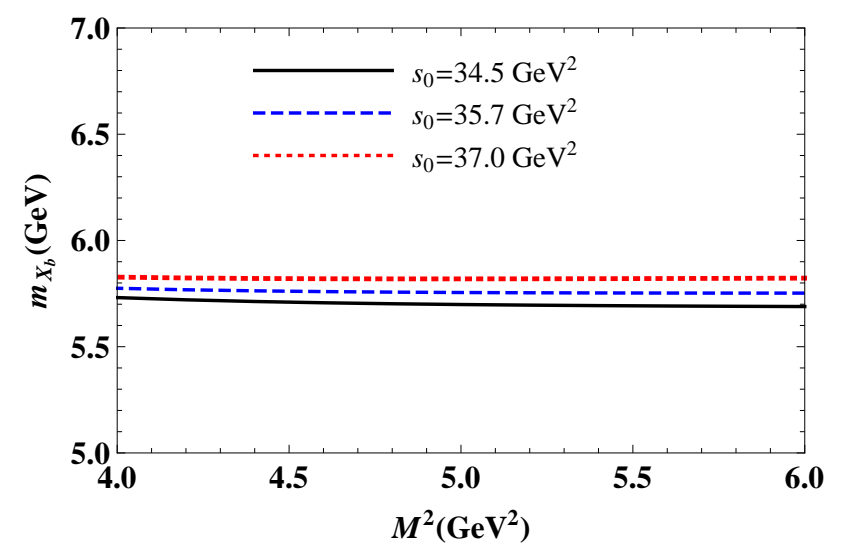

FIG. 1: The mass of the $X_{b}$ state versus the Borel parameter $M^{2}$.

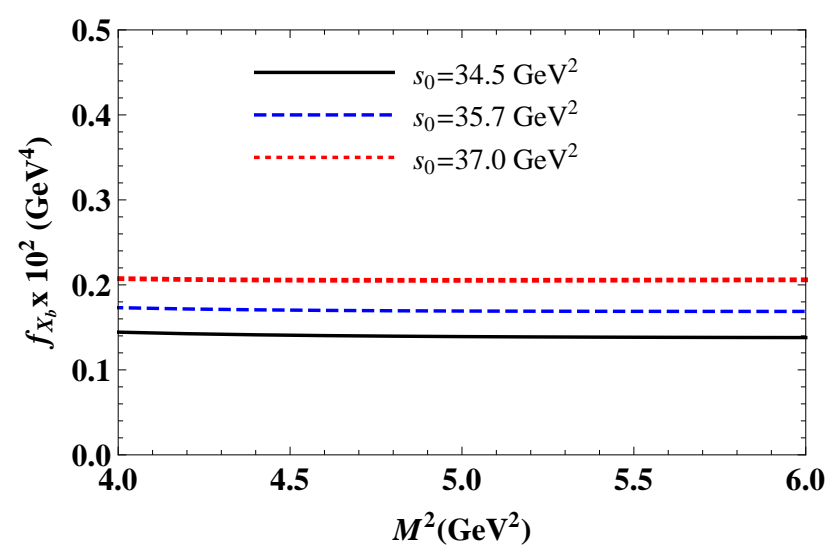

FIG. 2: The decay constant $f_{X_{b}}$ as a function the Borel parameter $M^{2}$.

As is seen, the values obtained for the mass and decay width of $X_{b}$ state by treating it as a meson molecule state, overshoot corresponding experimental data. This discrepancy is essential in the case of the mass, which is slightly less than the mass threshold of the $B \bar{K}$ system $\simeq 5778 \mathrm{MeV}$, and differs significantly from the experimental value of the D0 Collaboration. At the same time, the corresponding predictions obtained in Ref. [15, 16] using the diquark-antidiquark model for the $X_{b}$ state lead to a good agreement with experimental data of the D0 Collaboration.

The QCD sum rule predictions for the mass and decay width extracted in the present work by employing the molecule type interpolating current suffer from the large uncertainties. But such errors are inherent in the sum rule calculations, and are unavoidable part of the whole picture. The same conclusion is valid for the diquarkantidiquark current, as well. These two results have large overlap region making conclusive decision on the nature of the $X_{b}$ state rather problematic. Nevertheless, our results for the parameters of the $X_{b}$ state derived in the meson-molecule picture and their comparison

\begin{tabular}{|c|c|}
\hline \hline Parameters & Values \\
\hline \hline$m_{B_{s}}$ & $(5366.77 \pm 0.24) \mathrm{MeV}$ \\
$f_{B_{s}}$ & $(242 \pm 10) \mathrm{MeV}$ \\
$m_{\pi}$ & $139.57 \mathrm{MeV}$ \\
$f_{\pi}$ & $0.131 \mathrm{GeV}$ \\
$m_{b}$ & $(4.18 \pm 0.03) \mathrm{GeV}$ \\
$m_{s}$ & $(95 \pm 5) \mathrm{MeV}$ \\
$\langle\bar{q} q\rangle$ & $(-0.24 \pm 0.01)^{3} \mathrm{GeV}^{3}$ \\
$\langle\bar{s} s\rangle$ & $0.8\langle\bar{q} q\rangle$ \\
$m_{0}^{2}$ & $(0.8 \pm 0.1) \mathrm{GeV}^{2}$ \\
$\left\langle\frac{\alpha_{s} G^{2}}{\pi}\right\rangle$ & $(0.012 \pm 0.004) \mathrm{GeV}^{4}$ \\
$\left\langle g^{3} G^{3}\right\rangle$ & $(0.57 \pm 0.29) \mathrm{GeV}^{6}$ \\
\hline \hline
\end{tabular}

TABLE I: Input parameters.

with ones evaluated by applying the diquark-antidiquark model strengthen our confidence that if an exotic state $X_{b}$ with the parameters of the D0 Collaboration exists, the diquark-antidiquark form for its internal organization is more acceptable than configuration of a molecule built of the $B$ and $\bar{K}$ mesons.

In the present work we have performed QCD sum rule analysis of the exotic $X_{b}$ state by treating it as a molecule composed of the $B$ and $\bar{K}$ mesons. We have calculated the mass $m_{X_{b}}$ and width of the decay $\Gamma\left(X_{b} \rightarrow B_{s} \pi\right)$, and compared our results with the experimental data, as well as with predictions of the diquark-antidiquark picture. From the present analysis we conclude that the molecule model for the exotic $X_{b}$ state is less suitable than the diquark-antidiquark one to explain its parameters measured by the D0 Collaboration. Further investigations are required to clarify, first of all, the experimental situation emerged because of the information of the $\mathrm{LHCb}$ Collaboration. Theoretical studies have to be concentrated on preparing reliable models and computational schemes to treat such complicated many-quark systems like the exotic states.

\section{ACKNOWLEDGEMENTS}

The work of S. S. A. was supported by the TUBITAK grant 2221-"Fellowship Program For Visiting Scientists and Scientists on Sabbatical Leave". This work was also supported in part by TUBITAK under the grant no: $115 \mathrm{~F} 183$.

\section{Appendix: A}

In this appendix we have collected the results of our calculations of the spectral density

$$
\rho^{\mathrm{QCD}}(s)=\rho^{\mathrm{pert}}(s)+\sum_{k=3}^{8} \rho_{k}(s),
$$


used for evaluation of the $X_{b}$ meson mass $m_{X_{b}}$ and its decay constant $f_{X_{b}}$ from the QCD sum rule. In Eq. (A.1) by $\rho_{k}(s)$ we denote the nonperturbative contributions to $\rho^{\mathrm{QCD}}(s)$. In calculations we have neglected the masses of the $u$ and $d$ quarks and taken into account terms $\sim$ $m_{s}$. The explicit expressions for $\rho^{\text {pert }}(s)$ and $\rho_{k}(s)$ are presented below as integrals over the Feynman parameter $z$.

$$
\begin{aligned}
& \rho^{\text {pert }}(s)=\frac{1}{8192 \pi^{6}} \int_{0}^{a} \frac{d z z^{4}}{(1-z)^{3}}\left[m_{b}^{2}+s(z-1)\right]^{3}\left[m_{b}^{2}+3 s(z-1)\right], \\
& \rho_{3}(s)=\frac{3}{256 \pi^{4}} \int_{0}^{a} \frac{d z z^{2}}{(z-1)^{2}}\left[m_{b}^{2}+s(z-1)\right]\left\{-2\langle\bar{d} d\rangle m_{s}(1-z)\left[m_{b}^{2}+2 s(z-1)\right]-m_{b}^{3}\langle\bar{u} u\rangle+m_{b}^{2} m_{s}\langle\bar{s} s\rangle(1-z)\right. \\
& \left.-2 m_{s} s\langle\bar{s} s\rangle(z-1)^{2}+m_{b} s\langle\bar{u} u\rangle(1-z)\right\}, \\
& \rho_{4}(s)=\frac{1}{12288 \pi^{4}}\left\langle\alpha_{s} \frac{G^{2}}{\pi}\right\rangle \int_{0}^{a} \frac{d z z^{2}}{(1-z)^{3}}\left\{2 m_{b}^{4}\left(13 z^{2}-30 z+18\right)+3 m_{b}^{2} s(6-5 z)^{2}(z-1)+24 s^{2}(z-1)^{3}(2 z-3)\right\}, \\
& \rho_{5}(s)=\frac{m_{0}^{2}}{256 \pi^{4}} \int_{0}^{a} \frac{d z z}{(1-z)}\left\{3 m_{s}\langle\bar{d} d\rangle(z-1)\left[2 m_{b}^{2}+3 s(z-1)\right]-3 m_{b}^{3}\langle\bar{u} u\rangle-2 m_{b}^{2} m_{s}\langle\bar{s} s\rangle(z-1)-3 m_{b}\langle\bar{u} u\rangle s(z-1)\right. \\
& \rho_{6}(s)=\frac{1}{64 \pi^{4}} \int_{0}^{a} d z z\left\{\frac{z^{4}}{5120 \pi^{2}(1-z)^{3}}\left\langle g^{3} G^{3}\right\rangle\left[m_{b}^{2}(2 z+3)+s(z-1)(5 z-2)\right]-\frac{g^{2}}{27}\left[\langle\bar{u} u\rangle^{2}+\langle\bar{d} d\rangle^{2}+\langle\bar{s} s\rangle^{2}\right]\right. \\
& \left.\left.\times\left[2 m_{b}^{2}+3 s(z-1)\right]\right\}, \quad \rho_{8}^{2}\right\}, \quad \rho_{7}(s)=-\frac{1}{16 \pi^{2}} \int_{0}^{a} d z\left\{\frac{m_{b}^{2} z^{2}}{384(z-1)^{2}}\left\langle\alpha_{s} \frac{G^{2}}{\pi}\right\rangle^{2}\left[s \delta^{(1)}\left(s-m_{b}^{2} /(1-z)\right)+2 \delta\left(s-m_{b}^{2} /(1-z)\right)\right]\right. \\
& \left.+\frac{m_{0}^{2} m_{b} m_{s}}{16}\langle\bar{u} u\rangle[12\langle\bar{d} d\rangle-5\langle\bar{s} s\rangle] \delta\left(s-m_{b}^{2}\right)-m_{0}^{2}\langle\bar{d} d\rangle\langle\bar{s} s\rangle(z-1)\right\}, \\
& \left.\rho_{7}(s)=-\frac{G^{2}}{\pi}\right\rangle \int_{0}^{a} d z \frac{1}{(z-1)^{2}}\left\{2 m_{s}\langle\bar{d} d\rangle(5 z+1)(z-1)^{2}+m_{b}\langle\bar{u} u\rangle\left[z\left(2 z^{2}+7 z-14\right)+7\right]-6 m_{s}\langle\bar{s} s\rangle z(z-1)^{2}\right\},
\end{aligned}
$$

where $a=1-m_{b}^{2} / s$.

[1] S.-K. Choi et al. [Belle Collaboration], Phys. Rev. Lett. 91, 262001 (2003).

[2] V. M. Abazov et al. [D0 Collaboration], Phys. Rev. Lett. 93, 162002 (2004).

[3] D. Acosta et al. [CDF II Collaboration] Phys. Rev. Lett. 93, 072001 (2004).

[4] B. Aubert et al. [BaBar Collaboration], Phys. Rev. D 71, 071103 (2005).

[5] E. S. Swanson, Phys. Rept. 429, 243 (2006).

[6] E. Klempt and A. Zaitsev, Phys. Rept. 454, 1 (2007).
[7] S. Godfrey and S. L. Olsen, Ann. Rev. Nucl. Part. Sci. 58, 51 (2008).

[8] M. B. Voloshin, Prog. Part. Nucl. Phys. 61, 455 (2008).

[9] M. Nielsen, F. S. Navarra, and S. H. Lee, Phys. Rep. 497, 41 (2010).

[10] R. Faccini, A. Pilloni and A. D. Polosa, Mod. Phys. Lett. A 27, 1230025 (2012).

[11] A. Esposito, A. L. Guerrieri, F. Piccinini, A. Pilloni and A. D. Polosa, Int. J. Mod. Phys. A 30, 1530002 (2014).

[12] H.-X. Chen, W. Chen, X. Liu, and S.-L. Zhu, Arxiv: 
1601.02092 [hep-ph], 2016.

[13] V. M. Abazov et al. [D0 Collaboration], arXiv:1602.07588 [hep-ex].

[14] The LHCb Collaboration [LHCb Collaboration], LHCbCONF-2016-004, CERN-LHCb-CONF-2016-004.

[15] S. S. Agaev, K. Azizi and H. Sundu, Phys. Rev. D 93, 074024 (2016).

[16] S. S. Agaev, K. Azizi and H. Sundu, Phys. Rev. D 93, 114007 (2016).

[17] S. S. Agaev, K. Azizi and H. Sundu, Phys. Rev. D 93, 094006 (2016).

[18] Z. G. Wang, arXiv:1602.08711 [hep-ph].

[19] W. Wang and R. Zhu, arXiv:1602.08806 [hep-ph].

[20] W. Chen, H. X. Chen, X. Liu, T. G. Steele and S. L. Zhu, arXiv:1602.08916 [hep-ph].

[21] C. M. Zanetti, M. Nielsen and K. P. Khemchandani, arXiv:1602.09041 [hep-ph].

[22] J. M. Dias, K. P. Khemchandani, A. M. Torres, M. Nielsen and C. M. Zanetti, arXiv:1603.02249 [hep$\mathrm{ph}]$.

[23] Z. G. Wang, arXiv:1603.02498 [hep-ph].

[24] C. J. Xiao and D. Y. Chen, arXiv:1603.00228 [hep-ph].

[25] Y. R. Liu, X. Liu and S. L. Zhu, arXiv:1603.01131 [hep$\mathrm{ph}]$.
[26] X. G. He and P. Ko, arXiv:1603.02915 [hep-ph].

[27] Y. Jin and S. Y. Li, arXiv:1603.03250 [hep-ph].

[28] F. Stancu, arXiv:1603.03322 [hep-ph].

[29] T. J. Burns and E. S. Swanson, arXiv:1603.04366 [hep$\mathrm{ph}]$.

[30] L. Tang and C. F. Qiao, arXiv:1603.04761 [hep-ph].

[31] F. K. Guo, U. G. Mei?ner and B. S. Zou, arXiv:1603.06316 [hep-ph].

[32] Q. F. Lu and Y. B. Dong, arXiv:1603.06417 [hep-ph].

[33] A. Esposito, A. Pilloni and A. D. Polosa, arXiv:1603.07667 [hep-ph].

[34] M. Albaladejo, J. Nieves, E. Oset, Z. F. Sun and X. Liu, arXiv:1603.09230 [hep-ph].

[35] A. Ali, L. Maiani, A. D. Polosa and V. Riquer, arXiv:1604.01731 [hep-ph].

[36] S. S. Agaev, K. Azizi and H. Sundu, Phys. Rev. D 93, 074002 (2016).

[37] L. J. Reinders, H. Rubinstein and S. Yazaki, Phys. Rept. 127, 1 (1985).

[38] S. Narison, Nucl. Part. Phys. Proc. 270-272, 143 (2016).

[39] A. Bazavov et al. [Fermilab Lattice and MILC Collaborations], Phys. Rev. D 85, 114506 (2012). 